Particle Accelerators, 1995, Vol. 50, pp. 189-209

Reprints available directly from the publisher

Photocopying permitted by license only
(C) 1995 OPA (Overseas Publishers Association) Amsterdam B.V. Published under license by Gordon and Breach Science Publishers SA Printed in Singapore

\title{
SKEW CHROMATICITY
}

\author{
S. PEGGS and G.F. DELL \\ Relativistic Heavy Ion Collider, Brookhaven National Laboratory, \\ Upton, New York 11973, USA
}

(Received 2 February 1995; in final form 2 February 1995)

\begin{abstract}
The on-momentum description of linear coupling between horizontal and vertical betatron motion is extended to include off-momentum particles, introducing a vector quantity called the "skew chromaticity". This vector tends to be long in large superconducting storage rings, where it restricts the available working space in the tune plane, and modifies collective effect stability criteria. Skew chromaticity measurements at the Cornell Electron Storage Ring (CESR) and at the Fermilab Tevatron are reported, as well as tracking results from the Relativistic Heavy Ion Collider (RHIC). The observation of anomalous head-tail beam losses near the tune diagonal in the Tevatron are explained in terms of the extended theory, including modified criteria for head-tail stability. These results are confirmed in head-tail simulations. Sources of skew chromaticity are investigated.
\end{abstract}

KEY WORDS: Skew chromaticity, lattices, particle dynamics, storage rings

\section{INTRODUCTION}

This paper addresses the consequences of extending the standard on-momentum description of linear coupling to include off-momentum particles; an extension that introduces the concept of "skew chromaticity", in the form of a 2-D vector $\mathbf{k}$ which is embedded in a 3-D space.

There are two general areas of concern:

- A decoupled lattice that is well behaved on-momentum may be badly behaved offmomentum, inside the natural momentum spread of the beam. In the worst case the (fractional) design tunes must be moved away from the tune diagonal, reducing the free space available in the tune plane.

- Collective phenomena are often stabilised by adjusting the horizontal and vertical chromaticities. For example, theory and observation agree that the head-tail effect is stabilised by making the chromaticities slightly positive (above transition). Coupling must be taken into account close to the diagonal, and "chromaticities" must be replaced by "eigenchromaticities". 
No attempt is made to comprehensively survey the ways that momentum dependent coupling modifies collective stability behavior in general — not least because such a survey has not been performed. Rather, this paper illustrates the effects of skew chromaticity by deriving two modified criteria for head-tail stability. Experimental data from CESR and the Tevatron, and tracking data from RHIC, show that anomalous behavior is more likely in larger storage rings.

The first section below, On-momentum linear coupling, briefly summarises the standard linear coupling results in a formalism which is extended in the following section, Offmomentum linear coupling. This is followed, in turn, by a discussion of observed and simulated head-tail behaviour in Head-tail stability with momentum dependent coupling. Finally, the question Where does skew chromaticity come from? is addressed, with particular reference to RHIC.

\section{ON-MOMENTUM LINEAR COUPLING}

The natural dimensionless strength of a thin skew quadrupole is given by

$$
\alpha=\frac{\sqrt{\beta_{x} \beta_{y}}}{f}
$$

where $f$ is the focal length of the skew quad and $\beta_{x}$ and $\beta_{y}$ are its horizontal and vertical beta functions. It is convenient to go immediately to a $2-\mathrm{D}$ vector representation, where a skew quadrupole $i$ has a vector $\mathbf{q}_{i}$ with components along the orthogonal axes $\widehat{\mathbf{a}}$ and $\widehat{\mathbf{b}}$, given by

$$
\mathbf{q}_{i}=\frac{\alpha_{i}}{2 \pi}\left(\cos \left(\phi_{y}-\phi_{x}\right) \widehat{\mathbf{a}}:+: \sin \left(\phi_{y}-\phi_{x}\right) \cdot \widehat{\mathbf{b}}\right)
$$

where $\phi_{y}$ and $\phi_{x}$ are the vertical and horizontal betatron phases. When all skew quad vectors are summed together to give a resultant coupling vector $\mathbf{q}$, its length is equal to $\Delta Q_{\min }$, the closest approach of betatron eigentunes. ${ }^{1,2}$

$$
\Delta Q_{\min }=|q|=\left|\sum_{i} q_{i}\right|
$$

This minimum eigentune split is obtained when the two design tunes, $Q_{x}$ and $Q_{y}$, are made equal. The eigentunes $Q_{-}$and $Q_{+}$are given, for general design tunes, by

$$
Q_{ \pm}=\frac{1}{2}\left(Q_{x}+Q_{y}\right) \pm \frac{1}{2}|\mathbf{r}|
$$

where $\mathbf{r}$ is a 3-D vector given by

$$
\mathbf{r}=\mathbf{q}+\left(Q_{x}-Q_{y}\right) \widehat{\mathbf{c}}
$$


and $\widehat{\mathbf{c}}$ is a third orthogonal axis. The coupling vector $\mathbf{q}$ may be taken to be a 3-D vector, with one of its components always zero

$$
\mathbf{q} \cdot \widehat{\mathbf{c}} \equiv 0
$$

Note that Eqn. 4 explicitly guarantees that one eigentune is larger than the other:

$$
Q_{+} \geq Q
$$

as indicated mnemonically by the + or - subscript.

"Global decoupling" is routinely performed in most contemporary storage rings. ${ }^{2}$ Typically, two skew quadrupole families and one erect quadrupole family (or one tune variable) are empirically adjusted to reduce $\Delta Q_{\min }$ to as small a value as possible. In the language of Eqns. 4 and 5, this corresponds to making $\mathbf{r} \approx 0$.

"Local decoupling" is not a routine operational procedure in any storage ring, at the time of writing, although in the future it may become commonplace. ${ }^{3}$ It is analogous to closed orbit correction, in that diagnostic information is taken from many beam position monitors, in order to calculate optimum strengths for many independent correctors (in this case, skew quadrupoles). The goal, loosely speaking, is to make the eigenmodes at each position monitor as similar to pure horizontal and vertical motion as possible. As a rule of thumb, ${ }^{2}$ the typical angle of uncorrected eigenmode tilt is approximately given by $\Delta Q_{\min } /\left|Q_{x}-Q_{y}\right|$, the ratio of the minimum eigentune split to the design tune split. Thus, global decoupling is a useful precursor to local decoupling, much as $\Delta E / E$ errors are removed before correcting the closed orbit. Local decoupling is not discussed any further in this paper.

\section{OFF-MOMENTUM LINEAR COUPLING}

Eqn. 4 also describes the off-momentum eigentunes, $Q_{-}(\delta)$ and $Q_{+}(\delta)$, if the horizontal and vertical tunes are expanded to first order in $\delta=\Delta p / p$, the off-momentum parameter ${ }^{4,5}$

$$
\begin{aligned}
& Q_{x}(\delta)=Q_{x 0}+\chi_{x} \delta \\
& Q_{y}(\delta)=Q_{y 0}+\chi_{y} \delta
\end{aligned}
$$

The 3-D vector $r$ is also expanded, to become

$$
\mathbf{r}(\delta)=\mathbf{q}+\mathbf{k} \cdot \delta+\left[\left(Q_{x 0}-Q_{y 0}\right)+\left(\chi_{x}-\chi_{y}\right) \delta\right] \widehat{\mathbf{c}}
$$

These equations introduce the familiar "normal chromaticities" $\chi_{x}$ and $\chi_{y}$, and also introduce an important new quantity, the "skew chromaticity" vector $\mathbf{k}$. This vector, like $\mathbf{q}$, lies in the the $(a, b)$ plane, so that

$$
\mathbf{k} \widehat{\mathbf{c}} \equiv 0
$$

The skew chromaticity vector $\mathbf{k}$ has been measured at the Tevatron ${ }^{5}$ and at CESR ${ }^{6}$ and has been derived from tracking studies of RHIC. ${ }^{7}$ Results are shown in Table 1. The last column, which is discussed below, records the empirically observed closest approach of eigentunes. 
TABLE 1: Skew chromaticity and the approximate minimum eigentune split, as observed at three colliders. High luminosity optics were used in all cases. The RHIC $|\mathbf{k}|$ value depends on which random number seed is used to generate errors.

\begin{tabular}{lcc}
\hline machine & $|\mathbf{k}|$ & $\begin{array}{c}\Delta Q_{\text {min }} \\
\text { (observed) }\end{array}$ \\
\hline CESR & $0.5 \pm 0.5$ & 0.001 \\
Tevatron & $3.8 \pm 0.2$ & 0.003 \\
RHIC & 2.1 & \\
\hline
\end{tabular}

The further expansion of Eqns. 8, 9, and 10 to include higher order polynomial terms is straightforward. However, this paper is almost exclusively concerned with momentum dependent coupling that is linear in $\delta$.

\subsection{Eigenchromaticities}

The eigenchromaticities $\chi_{-}$and $\chi_{+}$are now defined as follows:

$$
\chi_{ \pm} \equiv \frac{d Q_{ \pm}}{d \delta}
$$

This leads to the simple result

$$
\chi_{ \pm}=\frac{1}{2}\left(\chi_{x}+\chi_{y}\right) \pm \frac{1}{2} \frac{\mathbf{r} . \mathbf{v}}{|\mathbf{r}|}
$$

where it is convenient to introduce the vector $\mathbf{v}$, such that

$$
\mathbf{v} \equiv \frac{d \mathbf{r}}{d \delta}
$$

Eqn. 13 is valid for any polynomial expansion of $\mathbf{r}$, but the situation is easiest to linear order in $\delta$, for then $\mathbf{v}$ is constant

$$
\mathbf{v}=\mathbf{k}+\left(\chi_{x}-\chi_{y}\right) \widehat{\mathbf{c}}
$$

in which case the vector $\mathbf{r}$ traces out a smoothly advancing straight line

$$
\mathbf{r}(\delta)=\mathbf{r}(0)+\mathbf{v} \cdot \delta
$$

as the off-momentum parameter is scanned.

\subsection{Examples in 2-D}

To gain some insight into what is going on, it is pedagogically useful to temporarily reduce the dimensionality of the model by considering the case when 


$$
\begin{aligned}
Q_{x 0} & =Q_{y 0} \equiv Q_{0} \\
\chi_{x} & =\chi_{y} \equiv \chi_{0}
\end{aligned}
$$

Substituting Eqn. 18 into Eqns. 10 and 15 yields

$$
\begin{aligned}
& \mathbf{r}=\mathbf{q}+\mathbf{k} \cdot \delta \\
& \mathbf{v}=\mathbf{k}
\end{aligned}
$$

thereby confining all vectors to the $(a, b)$ plane.

3.2.1 Perfect global decoupling First, consider the simplest possible non-trivial case, when a perfect job of global decoupling has been performed, so that

$$
\mathbf{q}=0
$$

Substitution into Eqns. 4 and 13 gives

$$
\begin{aligned}
& Q_{ \pm}=Q_{0} \pm \frac{1}{2}|k \cdot \delta| \\
& \chi_{ \pm}=\chi_{0} \pm \frac{1}{2}|k| \frac{\delta}{|\delta|}
\end{aligned}
$$

as shown in Figure 1 with $\chi_{0}=|\mathbf{k}|=4.0$. The eigentune split at a given value of $\delta$ is just

$$
Q_{+}-Q_{-}=|\mathbf{k} . \delta|
$$

This expression provides an intuitive feeling for what $\mathbf{k}$ does; if $|\mathbf{k}|=4$ and the rms momentum spread of the beam is $\sigma_{p} / p=10^{-3}$, then the eigentune split of a typical particle is 0.004 . This is significant when compared to the design tune separation of 0.01 employed at the Tevatron ${ }^{5}$ and at RHIC.
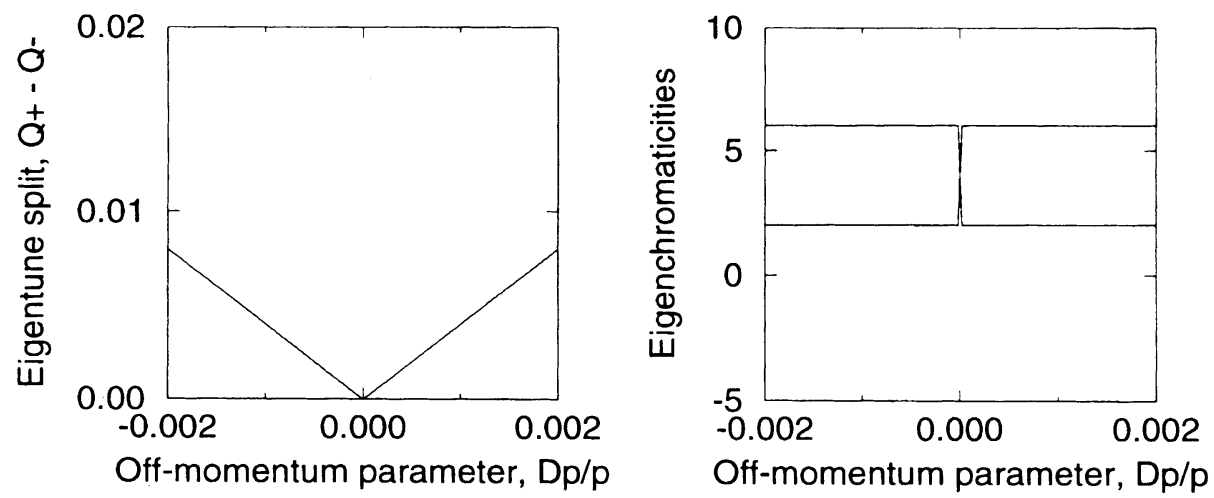

FIGURE 1: Eigentune split and eigenchromaticities when the design tunes are equal $\left(Q_{x 0}=Q_{y 0}\right)$, chromaticities are equal $\left(\chi_{x}=\chi_{y}=4.0\right)$, global decoupling has been perfectly performed $(\mathbf{q}=0)$, and the skew chromaticity is similar to the value in the Tevatron $(|\mathbf{k}|=4.0)$. 
Eqn. 24 also explains why it is difficult, in practice, to bring the eigentunes arbitrarily close together: even if global skew quadrupole correctors have been perfectly set, so that an on-momentum particle has $Q_{+}=Q_{-}$, most of the particles will still have a significant eigentune split. Since a spectrum analyser averages over all particles within a bunch, when the beams are excited, it is impossible to get the two peaks that appear there to be closer together than about

$$
\Delta Q_{\min }(\text { observed }) \approx|\mathbf{k}| \cdot \frac{\sigma_{p}}{p}
$$

It is interesting to note the coincidence that CESR, the Tevatron, and RHIC all have $\sigma_{p} / p \simeq 0.001$, although this number varies from shot to shot of coalesced beam in the Tevatron, and is not (yet) well known for RHIC. Thus, the prediction of Eqn. 25 is in good agreement with the last column of Table 1 .

3.2.2 Realistic global decoupling It is difficult during routine global decoupling to reduce $|\mathbf{q}|$ below $|\mathbf{k}| . \sigma_{p} / p$, because the primary diagnostic — eigentune measurement - is insensitive in this regime. Eqn. 21 does not, therefore, represent a plausible case in practice. Consider now the 2-D situation when $\mathbf{q} \neq 0$, and when either

$$
\widehat{\mathbf{q}} \cdot \widehat{\mathbf{k}}=0
$$

or

$$
\widehat{\mathbf{q}} \cdot \widehat{\mathbf{k}}=1
$$

The experimental measurement of the angle between the $\mathbf{q}$ and $\mathbf{k}$ vectors is possible, as reported for the Tevatron, ${ }^{5}$ if sufficient beam time is available.

Figures 2 and 3 show the behavior of the eigentunes and eigenchromaticities when $|\mathbf{q}|=0.003$, and when either Eqn. 26 or 27 applies. In the former case the main effect is broadening of the previously sharp features. In the latter case the features remain sharp, but the momentum at which the eigentunes are identical is shifted. According to Eqn. 4,
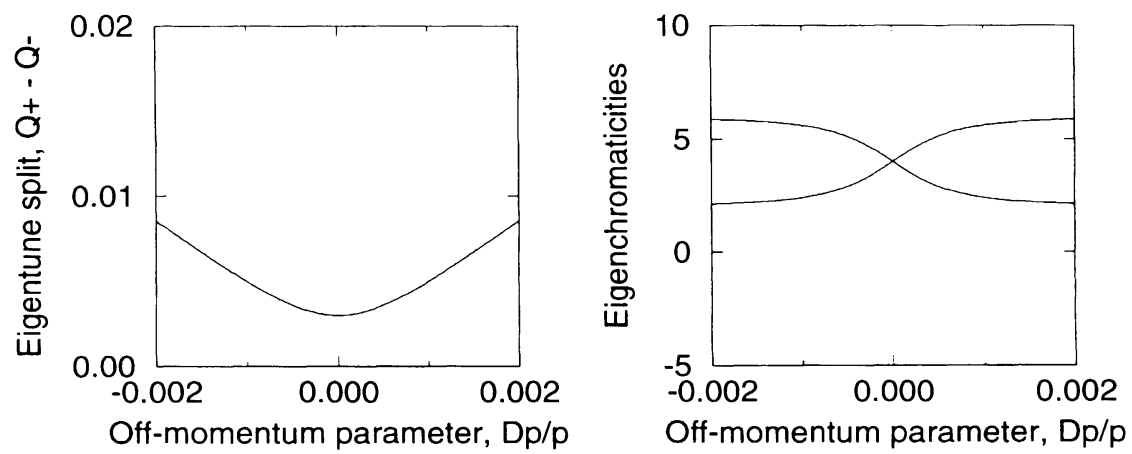

FIGURE 2: Eigentune split and eigenchromaticities with equal design tunes and chromaticities $\left(\chi_{x}=\chi_{y}=4.0\right)$, but with realistic global decoupling and skew chromaticity values $(|\mathbf{q}|=0.003,|\mathbf{k}|=4.0)$. In this case $\widehat{\mathbf{q}} . \widehat{\mathbf{k}}=0$, broadening the sharp features of Figure 1. 

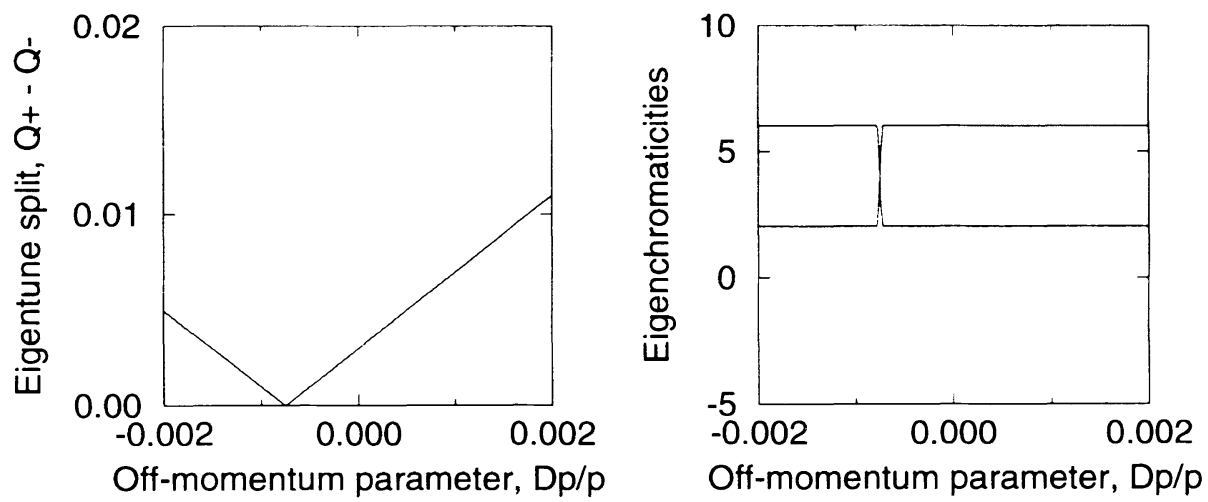

FIGURE 3: Eigentune split and eigenchromaticities with the same conditions as Figure 2, except that $\widehat{\mathbf{q}} \cdot \widehat{\mathbf{k}}=1$. This is identical to Figure 1, except that the sharp features are offset in momentum.

the closest approach of eigentunes occurs when $\mathbf{r}$ is shortest. This happens, in complete generality, when

$$
\mathbf{r . v}=0
$$

In the current 2-D context this reduces to

$$
\left(\mathbf{q}+\mathbf{k} \delta_{c}\right) \cdot \mathbf{k}=0
$$

with a solution

$$
\delta_{c}=-\frac{|\mathbf{q}|}{|\mathbf{k}|} \widehat{\mathbf{q}} \cdot \widehat{\mathbf{k}} \sim \frac{\sigma_{p}}{p}
$$

which is consistent with the figures.

\section{HEAD-TAIL STABILITY WITH MOMENTUM DEPENDENT COUPLING}

When coupling effects are unimportant, head-tail stability is assured if both chromaticities, $\chi_{x}$ and $\chi_{y}$, are slightly positive. ${ }^{8,9}$ It seems reasonable to make a "strong conjecture", that head-tail stability is only guaranteed in the presence of coupling if the eigenchromaticities are positive for all momenta within the beam. However, it is also reasonable to make a "weak conjecture", that it is only necessary for on-momentum particles to have positive eigenchromaticities. This is close to (but more convenient than) speculating that it is the average eigenchromaticity over one synchrotron period that is the fundamental quantity. This section investigates these conjectures, with reference to experimental data from the Tevatron and numerical data from a simple simulation of the head-tail effect.

First, we derive strong new criteria for $\chi_{x}$ and $\chi_{y}$ which guarantee that the eigenchromaticities are always positive, not only for all momenta, but also for all skew quadrupole and erect quadrupole settings. 


\section{$4.1 \quad$ Extreme eigenchromaticities}

According to Eqn. 13, the extreme values of $\chi_{+}$and $\chi_{-}$(with respect to changes in $\delta, \mathbf{q}, Q_{x 0}$ and $Q_{y 0}$ ) occur when

$$
\frac{\mathbf{r . v}}{|\mathbf{r}|}= \pm|\mathbf{v}|
$$

that is, when $\mathbf{r}$ and $\mathbf{v}$ are collinear. Collinearity is always acheived when

$$
\mathbf{r}(\delta)=\mathbf{v} \delta
$$

which occurs, for example, when

$$
\begin{aligned}
\mathbf{q} & =0 \\
Q_{x 0} & =Q_{y 0}
\end{aligned}
$$

This corresponds, physically, to a machine in which a perfect job of global decoupling has been performed, and the design tunes have been set equal. The values of the extreme eigenchromaticities are found by substitution into Eqn. 13, yielding

$$
\chi_{ \pm \text {extreme }}=\frac{1}{2}\left(\chi_{x}+\chi_{y}\right) \pm \frac{1}{2} \sqrt{k^{2}+\left(\chi_{x}-\chi_{y}\right)^{2}}
$$

Insisting that both of the extreme eigenchromaticities are positive leads to the criteria

$$
\begin{aligned}
\chi_{x}+\chi_{y} & >0 \\
4 \chi_{x} \chi_{y} & >\mathbf{k}^{2}
\end{aligned}
$$

These criteria are new and strong: if true, neither eigenchromaticity can ever become negative, for all momenta and for all erect and skew quadrupole settings. The criteria are sufficient (but probably not necessary) for positive eigenchromaticities in any particular lattice configuration. In order for both criteria to be met, $\chi_{x}$ and $\chi_{y}$ must be positive, even when $k=0$, thereby recovering the standard uncoupled head-tail result.

\subsection{Tevatron observations and measurements}

During the 1989-1990 Tevatron collider run, the operators observed that high intensity bunches $\left(N_{\text {bunch }}>6 \times 10^{10}\right)$ would occasionally go unstable if the machine ran close to the coupling resonance $\left(Q_{+}-Q_{-} \lesssim 0.005\right)$. Sometimes beam losses were spontaneous. Sometimes they were initiated by human intervention - for example, when the operators periodically compensated for persistent current drifts by returning the tunes and the chromaticities to their optimum values. A preliminary theoretical analysis ${ }^{4}$ indicated that head-tail instability could arise as the working point approached the diagonal, but the operators reported that, to the contrary, beam loss often occurred when the tunes were being separated. How could theory and observation be reconciled? 
TABLE 2: Tevatron coupling parameters, as found by fitting several tune versus momentum curves, for various lattice configurations. Eigentunes were derived from spectrum analyser peaks that were externally excited. The central momentum was varied by changing the RF frequency.

\begin{tabular}{cc}
\hline parameter & value \\
\hline$|\mathbf{q}|$ & $0.0032 \pm 0.0008$ \\
$|\mathbf{k}|$ & $3.8 \pm 0.2$ \\
$\widehat{\mathbf{k}} \cdot \widehat{q}$ & $\approx 1$ \\
\hline
\end{tabular}
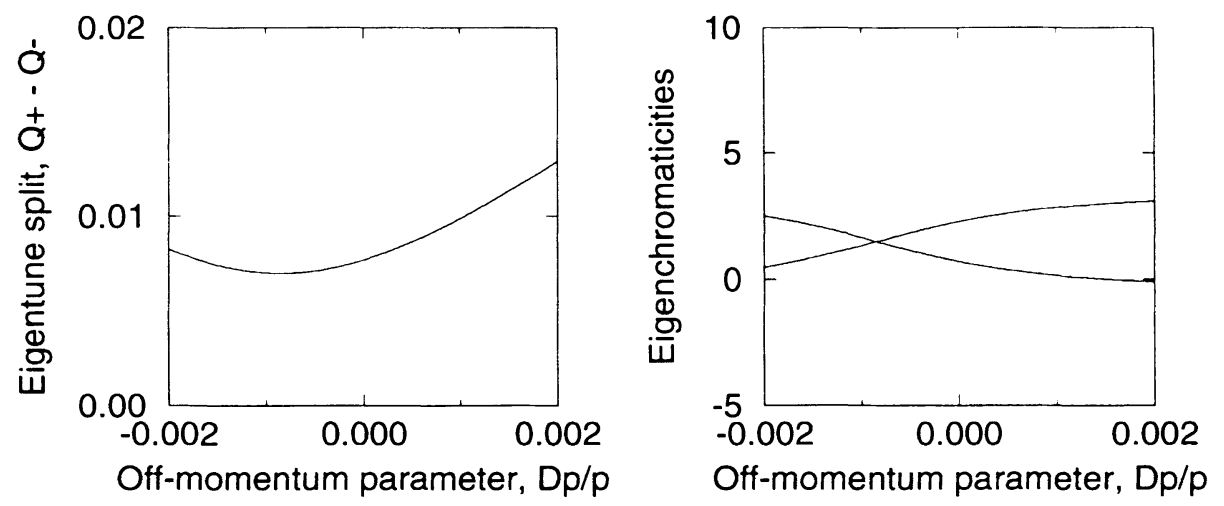

FIGURE 4: Tevatron eigentune split and eigenchromaticities when the design chromaticities were equal $\left(\chi_{x}=\right.$ $\left.\chi_{y}=1.5\right)$, and the design tunes were 0.007 apart $\left(Q_{x 0}=0.425, Q_{y 0}=0.418\right)$. The beam was stable.
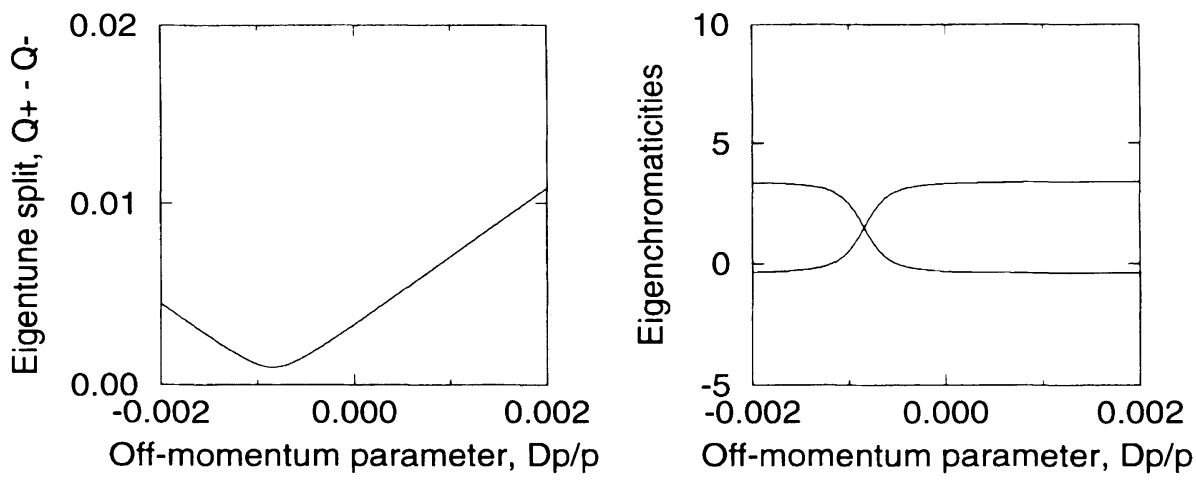

FIGURE 5: Tevatron eigentune split and eigenchromaticities under the same conditions as in Figure 4, except that the design tunes were brought together to be only 0.001 apart $\left(Q_{x 0}=0.419, Q_{y 0}=0.418\right)$. Beam loss was observed under these conditions. One eigenchromaticity was negative for all positive momentum offsets. 
Detailed results from dedicated beam studies of "Head-tail stability and linear coupling in the Tevatron" have been published elsewhere. ${ }^{5}$ Stability was investigated for different lattice configurations - different settings of the design tunes and chromaticities, $Q_{x 0}, Q_{y 0}, \chi_{x}$, and $\chi_{y}$. The skew quadrupole family strengths were fixed during the experiments, after they were initially set by a round of global decoupling. Experimental curves of tune versus momentum, for various lattice configurations, were all well fit by the parameters listed in Table 2. Entirely different behavior was observed between the case when the chromaticities were set equal, and the case when they were set grossly different.

When the chromaticities were both set equal to $\chi_{0}$, the horizontal design tune $Q_{x 0}$ was scanned across the tune diagonal, in a search for head-tail induced beam loss. This was repeated for $\chi_{0}=4,3,2$, and 1 , with significant beam loss observed as the diagonal was approached for the last two values. This is consistent with the criterion of Eqn. 37, which predicts unequivocal stability with equal chromaticities when

$$
\chi_{0}>1.9 \pm 0.1
$$

Figures 4 and 5 show the eigentune split and the eigenchromaticities as functions of momentum, when $\chi_{0}=1.5$, for two different design tune splits. Both eigenchromaticities are above zero for all momentum offsets when the design tunes are 0.007 apart. Conversely, one eigenchromaticity is negative for all positive momenta when $Q_{x 0}$ and $Q_{y 0}$ are only 0.001 apart.

The behavior of the Tevatron was quite different when the chromaticities were set to $\left(\chi_{x}, \chi_{y}\right)=(8,-3)$. (These superficially bizarre chromaticity values were not chosen merely out of intellectual curiosity: persistent current drifts in the Tevatron force the horizontal and vertical chromaticities to move in opposite directions. These values are not unrealistic). In this case total beam loss was observed as the design tunes retreated from the diagonal. This cannot be understood in terms of the (sufficient but not necessary) stability criteria of Eqns. 37 and 38. Empirical insight is given by Figures 6 and 7,
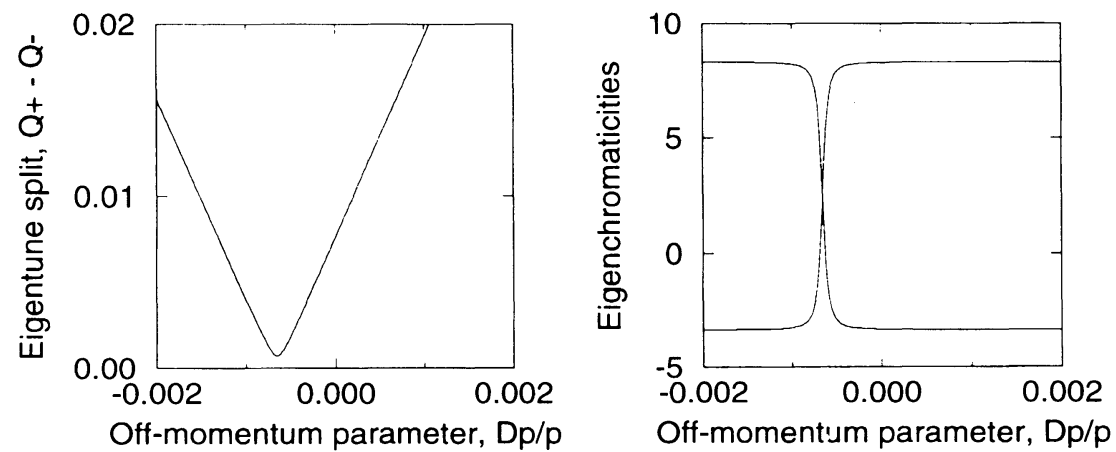

FIGURE 6: Tevatron eigentune split and eigenchromaticities when the design chromaticities were very unequal $\left(\chi_{x}=8.0, \chi_{y}=-3.0\right)$. Such values can be reached after a long period of persistent current drift. In this case the design tunes were 0.007 apart $\left(Q_{x 0}=0.425, Q_{y 0}=0.418\right)$. The beam was unstable - total beam loss was observed - due to the very negative eigenchromaticities. 

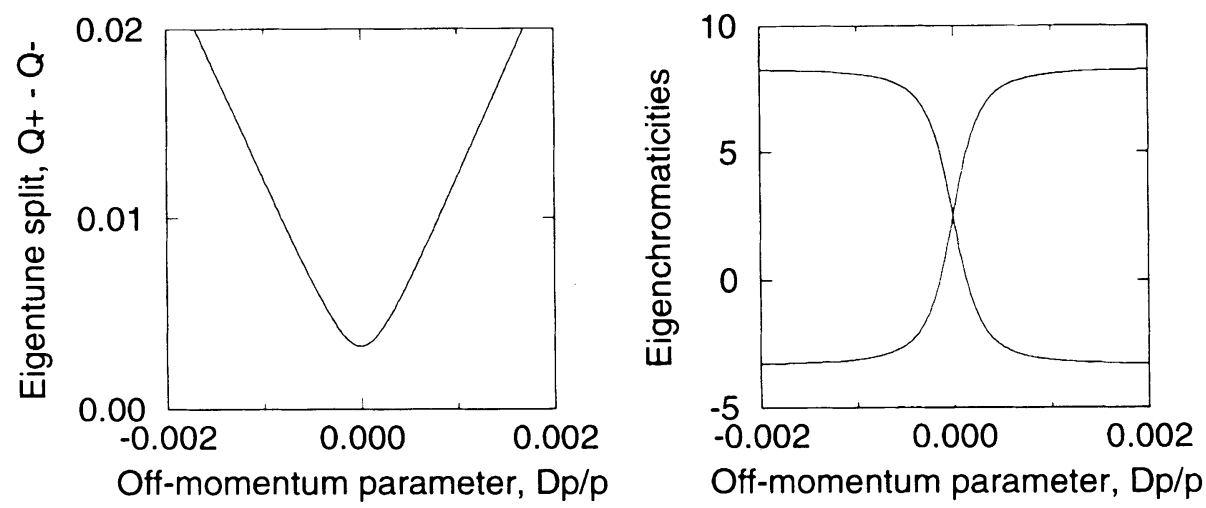

FIGURE 7: Tevatron eigentune split and eigenchromaticities under the same conditions as in Figure 6, except that the design tunes were only 0.001 apart $\left(Q_{x 0}=0.417, Q_{y 0}=0.418\right)$. It is remarkable that the beam was stable. Both eigenchromaticities were positive for $\delta \approx 0$.

which again plot the eigentune split and the eigenchromaticities when the design tunes are 0.007 and 0.001 apart. In this case, one of the two eigenchromaticities is negative for all positive momentum offsets when the tunes are separated by 0.007 , but both are positive when the tunes are only 0.001 apart, at least in a small vicinity around zero momentum offset.

These experimental results support the "weak conjecture" — that only the on-momentum eigenchromaticities are important in determining head-tail stability.

\subsection{A simple two macroparticle head-tail model}

Various authors have reported analytical and simulation results for a linearised head-tail model, in which a bunch of total population $N$ is broken into two macroparticles. ${ }^{8-11}$

When macroparticle 2 trails macroparticle 1 by a distance $s$, it is accelerated horizontally and vertically by the transverse wake field

$$
\begin{aligned}
\frac{d^{2} x_{2}}{d t^{2}} & =\frac{N e^{2}}{2 m \gamma} W_{1}(s) x_{1} \\
\frac{d^{2} y_{2}}{d t^{2}} & =\frac{N e^{2}}{2 m \gamma} W_{1}(s) y_{1}
\end{aligned}
$$

where $\gamma$ is the Lorentz factor and $m$ is the mass of an individual particle. The model becomes linear, and hence analytically soluble, if the wake force $W_{1}$ is (unrealistically) taken to be a step function:

$$
\begin{array}{ll}
W_{1}(s)=W_{1} & s>0 \\
W_{1}(s)=0 & s<0
\end{array}
$$


Implicitly assuming that the horizontal and vertical betatron motion is linear and uncoupled, and taking the longitudinal motion to be inexorable, strong head-tail stability is assured if

$$
\Upsilon \equiv \frac{N e^{2} W_{1} T_{s}}{16 \pi f_{0}^{2} Q m \gamma}<2
$$

where $T_{s}$ is the synchrotron period in turns, $f_{0}[\mathrm{~Hz}]$ is the revolution frequency, and $Q$ is the total betatron tune (including the integer part). This stability criterion does not contain the chromaticity, which is taken to be zero. Note that $\Upsilon$ is proportional to $T_{s}$, showing that a high synchrotron tune offers insurance against instability.

Motion of the two macroparticles can be decomposed into two eigenmodes in each uncoupled plane. The pure "+" mode ("-" mode) occurs when the macroparticles oscillate in phase (out of phase). When a modest chromaticity $\chi$ is introduced in a single plane, the head-tail growth rates per turn for the eigenmodes of a low intensity bunch are

$$
\tau_{ \pm}^{-1}=\mp \frac{4 \Upsilon}{\pi} \chi \widehat{\delta} \quad[1 / \text { turn }]
$$

where $\widehat{\delta}$ is the momentum amplitude of a macroparticle. This equation implies that either the "+" mode grows and the center of charge amplitude increases exponentially, or the "-" mode grows and the beam size increases, eventually leading to beam loss no matter what the chromaticity. Fortunately, the two macroparticle model overestimates $\tau_{-}^{-1}$, and in practice both modes are stabilised, above transition, by a slightly positive chromaticity. ${ }^{8}$

Figure 8 shows what happens to the horizontal amplitude growth rate of simulated motion, when the horizontal chromaticity is not modest, but the betatron motion remains uncoupled. The dashed line is an empirical fit

$$
\tau_{ \pm}^{-1}=\mp \frac{4 \Upsilon}{\pi T_{s}} J_{1}\left(2 \chi \widehat{\delta} T_{s}\right) \quad[1 / \text { turn }]
$$

which reduces to Eqn. 44 in the limit of small chromaticity. The empirical fit is well synchronised with the observed oscillations in the amplitude rise time, but the oscillation amplitudes are only in fair agreement.

Finally, Figure 9 shows what happens when skew quadrupoles and skew chromaticity introduce momentum dependent coupling between the horizontal and vertical betatron motion, with the Tevatron parameters of Table 2 . The simulated region of stability is shifted to the right, with the amplitude growth rate going negative at $\chi \approx 2.0$. This is in remarkably good agreement with the analytically predicted criterion of Eqn. 38, which itself comes from the new stability criteria of Eqns. 36 and 37. It is worth commenting that clean simulation data, as shown here, are only obtained when the initial macroparticle conditions correspond to a pure local eigenmode of the coupled system.

\section{WHERE DOES SKEW CHROMATICITY COME FROM?}

The total skew chromaticity vector $\mathbf{k}$ is the sum of vectors $k_{i}$,

$$
\mathbf{k}=\sum_{i} \mathbf{k}_{i}
$$




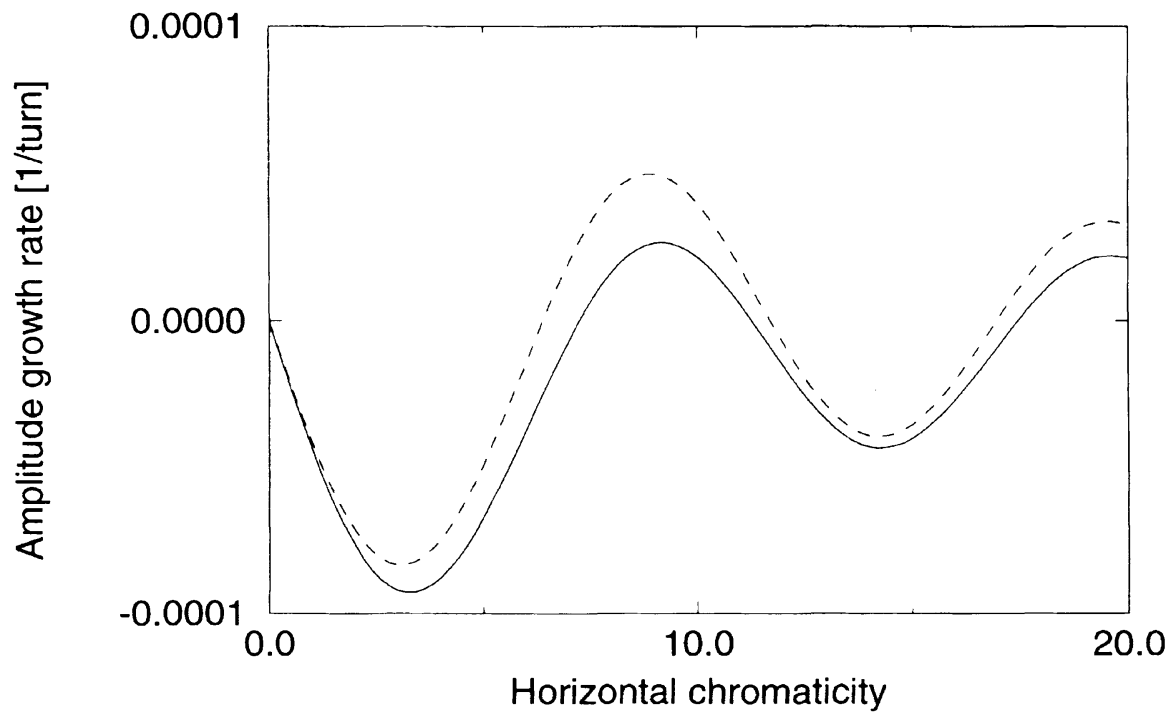

FIGURE 8: The horizontal amplitude rise time as a function of the horizontal chromaticity, as observed by simulation (solid line). The synchrotron period is $T_{s}=300$ turns, $\widehat{\delta}=0.001$, and there is no linear coupling. Empirical theory (dashed line) and observation are in fair agreement.

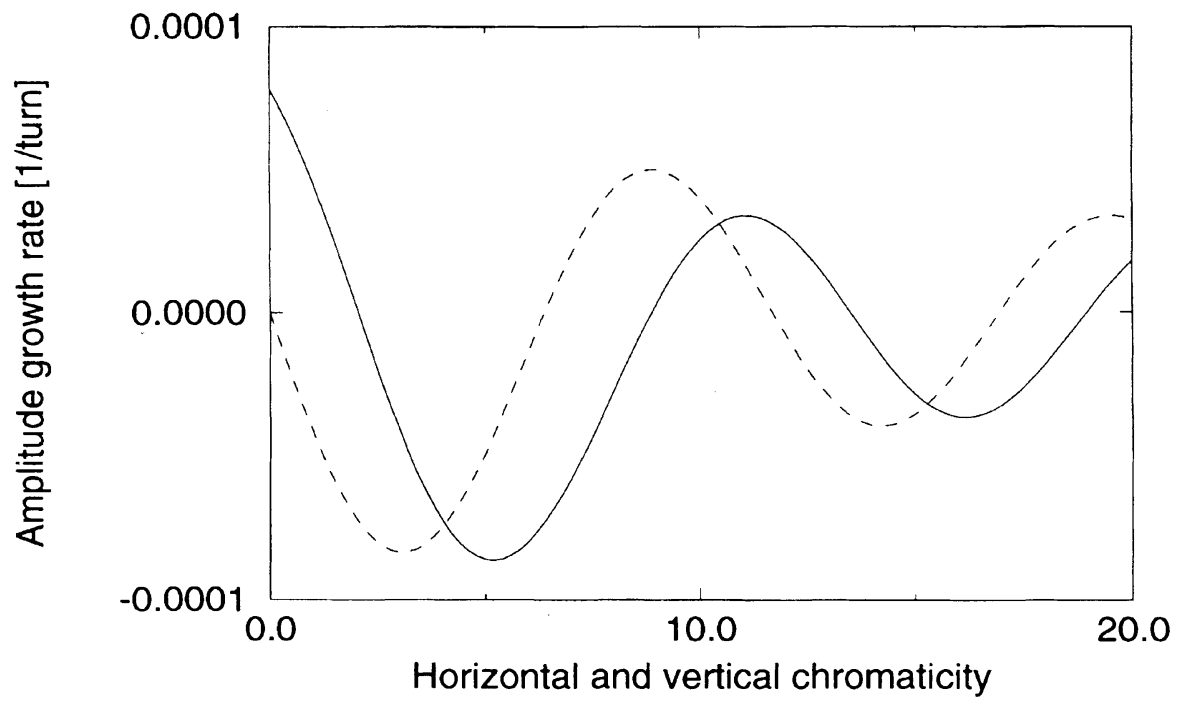

FIGURE 9: The simulated amplitude rise time for one local eigenmode as a function of equal horizontal and vertical chromaticities, with the same conditions as Figure 8, except that linear coupling is present with the Tevatron-like parameters of Table 2 . The design tunes are equal. 
where each component $\mathbf{k}_{i}$ is the first differential of a skew quad vector

$$
\mathbf{k}_{i}=\frac{d \mathbf{q}_{i}}{d \delta}
$$

Substitution of Eqns. 1 and 2 into Eqn. 47 shows that, in general, differentials of $\beta_{i}$ and $\phi_{i}$ both contribute to $\mathbf{k}$. In practice, however, the dominant contribution is from the variation of $f$, the skew quadrupole strength, with respect to momentum. This comes mainly from chromatic feed down in sextupoles - from horizontal dispersion in skew sextupoles and from vertical dispersion in normal sextupoles.

For example, a thin skew sextupole element of strength $A_{2}$ gives a vertical angular kick of

$$
\Delta y^{\prime}=A_{2} x_{\text {tot }}^{2}
$$

where the total displacement in the horizontal plane is

$$
x_{\text {tot }}=x+\eta_{x} \delta
$$

while $x$ is the betatron oscillation displacement and $\eta_{x}$ is the horizontal dispersion. This causes the skew sextupole to have a skew quadrupole component with a focal strength

$$
\frac{1}{f}=2 A_{2} \eta_{x} \delta
$$

that is linear in the off-momentum parameter. So, in turn,

$$
\left|\mathbf{k}_{i}\right|=\frac{A_{2}}{\pi} \eta_{x} \sqrt{\beta_{x} \beta_{y}}
$$

Similarly, a normal sextupole of integrated strength $B_{2}$ with a vertical dispersion of $\eta_{y}$ has a skew chromaticity element strength of

$$
\left|\mathbf{k}_{i}\right|=\frac{B_{2}}{\pi} \eta_{y} \sqrt{\beta_{x} \beta_{y}}
$$

Most storage rings are designed to lie in a horizontal plane, so that the idealised vertical dispersion is zero. Although there are strong chromaticity correction sextupoles, skew sextupoles are not present by design. Hence, there are usually only accidental sources of skew chromaticity. The question therefore becomes: "In the presence of realistic features and errors, how strong are the skew sextupole elements, and how large is the vertical dispersion at chromaticity sextupoles?" The following subsections attempt to answer this question, with reference to a generic FODO lattice, and to a case study of RHIC.

\subsection{FODO lattices}

RHIC, like most storage rings, can be conceptually broken into FODO cell arcs and special purpose interaction or utility regions. Chromaticity correction is performed by two families of chromaticity sextupoles in the FODO cells, labeled $F$ and $D$, according to whether the 
neighboring quadrupole is horizontally focusing or defocusing. All of the sextupoles in one family have, at least approximately, the same optical functions. It is therefore interesting to study the simple example of a pure FODO cell lattice, with no interaction or utility regions, in order to get a quantitative feeling for the parameters involved, and to see how they scale.

The simplest possible FODO half cell consists of a thin quadrupole next to a thin sextupole, immediately followed by a long dipole. If the phase advance per half cell is $\phi_{1 / 2}$ in both planes, then the three natural control parameters are the length of the dipole (and the half cell) $L$, the bending radius of the dipole (and the radius of the accelerator) $R$, and $s$, where

$$
s \equiv \sin \left(\phi_{1 / 2}\right)
$$

For example, the maximum and minimum optical functions are

$$
\begin{aligned}
& \beta_{\max }=\frac{L}{s} \sqrt{\frac{1+s}{1-s}} \\
& \beta_{\min }=\frac{L}{s} \sqrt{\frac{1-s}{1+s}} \\
& \eta_{\max }=\frac{L^{2}}{R} \frac{2+s}{2 s^{2}} \\
& \eta_{\min }=\frac{L^{2}}{R} \frac{2-s}{2 s^{2}}
\end{aligned}
$$

Similarly, the focal strength of the quadrupoles and the geometric strength of the $F$ and $D$ sextupoles, $B_{2 F}$ and $B_{2 D}$, are

$$
\begin{aligned}
& \frac{1}{|f|}=\frac{2 s}{L} \\
& B_{2 F}=\frac{R}{L^{3}} \frac{2 s^{3}}{2+s} \\
& B_{2 D}=\frac{R}{L^{3}} \frac{-2 s^{3}}{2-s}
\end{aligned}
$$

where the sextupoles have been set to exactly cancel the natural chromaticity of the FODO cells. Note, finally, that there are

$$
N=\frac{2 \pi R}{L}
$$

half cells in a lattice that closes properly, since there is a bend angle of $L / R$ per dipole.

A FODO approximation to a real machine significantly underestimates the strengths of the chromaticity sextupoles (and hence also underestimates skew chromaticity effects) by as much as an order of magnitude. This is not only because the interaction region optics 
TABLE 3: FODO parameters for the equivalent lattice representing RHIC.

\begin{tabular}{lccc}
\hline half cell parameter & symbol & units & value \\
\hline length & $L$ & {$[\mathrm{~m}]$} & 14.9 \\
radius & $R$ & {$[\mathrm{~m}]$} & 358.2 \\
phase advance & $\phi_{1 / 2}$ & {$[\mathrm{deg}]$} & 42.1 \\
& $N$ & & 151.1 \\
& $s$ & & 0.671 \\
\hline
\end{tabular}

contribute a large part of the natural chromaticity in luminosity optics, but also because, in practice, thin sextupoles cannot be placed right next to thin quadrupoles. It is therefore important to remember, below, that the use of equivalent FODO cell parameters leads to an underestimate of the severity of the situation.

\subsection{The equivalent FODO model of RHIC}

Table 3 lists the optimum parameters for an equivalent FODO cell model of RHIC. The half cell phase advance $\phi_{1 / 2}$ has been set equal to the average of the (slightly different) horizontal and vertical half cell phase advances in the arcs, while the half cell length and radius, $L$ and $R$, have been adjusted to best match the maximum and minimum optical functions in the actual matched arc cell.

Table 4 shows how well this simple FODO model matches real RHIC parameters. The optical functions are matched to within a few per cent, and the number of $F$ and $D$ family sextupoles is only slightly overestimated in the FODO equivalent lattice. As expected for reasons discussed above, the FODO lattice seriously underestimates the strength of the sextupole families in the real RHIC luminosity optics, by a factor of 3.7 for the $F$ family and 4.3 for the $D$ family.

TABLE 4: Equivalent FODO lattice parameters for RHIC, compared to the actual values in luminosity optics, when $\beta^{*}=1 \mathrm{~m}$ at two of the six interaction points.

\begin{tabular}{lcccc}
\hline parameter & symbol & units & FODO value & actual value \\
\hline Maximum horizontal $\beta$ & $\beta_{x F}$ & {$[\mathrm{~m}]$} & 50.1 & 48.6 \\
Minimum vertical $\beta$ & $\beta_{y F}$ & {$[\mathrm{~m}]$} & 9.9 & 9.9 \\
Minimum horizontal $\beta$ & $\beta_{x D}$ & {$[\mathrm{~m}]$} & 9.9 & 10.7 \\
Maximum vertical $\beta$ & $\beta_{y D}$ & {$[\mathrm{~m}]$} & 50.1 & 47.2 \\
Maximum dispersion & $\eta_{x F}$ & {$[\mathrm{~m}]$} & 1.84 & 1.83 \\
Minimum dispersion & $\eta_{x}$ & {$[\mathrm{~m}]$} & 0.91 & 0.92 \\
Number of $F$ sextupoles & $N_{F}$ & & 75.6 & 72 \\
Number of $D$ sextupoles & $N_{D}$ & & 75.6 & 72 \\
Strength of $F$ sextupoles & $B_{2 F}$ & {$\left[\mathrm{~m}^{-2}\right]$} & 0.0245 & 0.0905 \\
Strength of D sextupoles & $B_{2 D}$ & {$\left[\mathrm{~m}^{-2}\right]$} & -0.0492 & -0.2094 \\
\hline
\end{tabular}




\subsection{Rolled chromaticity sextupoles}

If a chromaticity sextupole with strength $B_{2}$ has been accidentally rolled by a small angle $\theta$, it acquires a skew sextupole component of strength

$$
A_{2}=3 \theta B_{2}
$$

It follows from Eqn. 51 that

$$
\left|\mathbf{k}_{i}\right|=\frac{3 \theta}{\pi} G_{i}
$$

where it is convenient to introduce the factor $G_{i}$ given by

$$
G_{i} \equiv B_{2} \eta_{x} \sqrt{\beta_{x} \beta_{y}}
$$

A surprising result is that the values $G_{F}$ and $G_{D}$ at $F$ and $D$ locations in a FODO lattice are simply

$$
G_{F}=-G_{D}=1
$$

A better approximation for $G_{F}$ and $G_{D}$ in a storage ring like RHIC (although still an underestimate) is

$$
G_{F} \approx-G_{D} \lesssim \frac{\chi_{\text {natural }}}{\chi_{\text {arc }}}
$$

where $\chi_{\text {natural }}$ is the total natural chromaticity, including the interaction regions, while $\chi_{\text {arc }}$ is the natural chromaticity from the regular arc cells alone. According to the discussion above,

$$
G_{F} \approx-G_{D} \approx 4
$$

for the particular case of the RHIC luminosity lattice.

In a simple FODO lattice where the chromaticity sextupoles have a random roll, the rms length of the total skew chromaticity vector is just

$$
|\mathbf{k}|=\frac{3 \sqrt{N}}{\pi}<\theta^{2}>^{1 / 2}
$$

where $N$ is the number of half cells. For example, if the 151.1 sextupoles in the FODO equivalent lattice of RHIC have a random roll angle of 1 milliradian, then it is reasonable to expect a skew chromaticity vector of length

$$
|\mathbf{k}| \approx 0.012
$$

The smallness of this value shows that rolled chromaticity sextupoles are not expected to be a major source of skew chromaticity in RHIC, even if realistic values for $G_{F}$ and $G_{D}$ are used. 


\subsection{Vertical dispersion in chromaticity sextupoles}

Eqn. 52 describes the skew chromaticity element strength of a chromaticity sextupole when vertical dispersion is present. It is convenient to rewrite this equation, for a typical element in the presence of random errors, with the aid of Eqn. 65. It becomes

$$
\left|\mathbf{k}_{i}\right|=\frac{G_{i}}{\pi} \frac{\eta_{y}^{2}>^{1 / 2}}{\eta_{x}}
$$

The root mean square vertical dispersion $<\eta_{y}^{2}>^{1 / 2}$ is caused by error effects, while the horizontal dispersion $\eta_{x}$ is the design value. It is now useful to introduce the optical pseudo-invariant

$$
u \equiv \frac{\eta_{x}}{\sqrt{\beta_{x}}}
$$

that describes the matched dispersion function in the regular arc of a storage ring. This quantity is only approximately constant: its values at the $F$ and $D$ quads in the RHIC equivalent FODO lattice are 0.260 and 0.289 , respectively. The pseudo-invariant is used to scale the rms vertical dispersion at different locations in a storage ring, through

$$
<\eta_{y}^{2}>^{1 / 2} \approx \epsilon u \sqrt{\beta_{y}}
$$

where the quantity $\epsilon$ measures the degree of vertical dispersion present: $\epsilon=0$ in an ideal ring, but $\epsilon \approx 1$ in a ring rife with vertical dispersion. Substitution into Eqn. 70 yields

$$
\left|\mathbf{k}_{i}\right| \approx \frac{\epsilon G_{i}}{\pi} \sqrt{\frac{\beta_{y}}{\beta_{x}}}
$$

which shows that it is the sextupoles at $D$ locations that dominate the generation of skew chromaticity, since $\beta_{y D} \gg \beta_{x D}$.

Neglecting the contribution from the $F$ sextupoles, the total skew chromaticity vector in a lattice with $N_{D} D$ family sextupoles is typically

$$
|\mathbf{k}| \approx \frac{\epsilon G_{D} \sqrt{N_{D}}}{\pi} \sqrt{\frac{\beta_{y D}}{\beta_{x D}}}
$$

For example, the $72 D$ sextupoles in RHIC generate a total skew chromaticity of

$$
|\mathbf{k}| \approx 5.7\left|G_{D}\right| \epsilon
$$

where optical values from Table 4 have been used. This shows that vertical dispersion in chromaticity sextupoles is a potent source of skew chromaticity in RHIC - especially when the realistic value $G_{D} \approx-4$ is used. Tracking simulations show that $\epsilon \approx 0.05$ to 0.10 if there is no deliberate attempt to control vertical dispersion in RHIC, dominating the observed value of $|\mathbf{k}| \approx 2.1$. At the time of writing, vertical dispersion correction schemes are being evaluated for application in RHIC. 
TABLE 5: Dominant sources of SYSTEMATIC skew sextupole kicks in RHIC.

\begin{tabular}{lrrrrrr}
\hline Magnet & Count & $\begin{array}{r}\text { Skew } \\
\text { sext. } \\
\text { strength }\end{array}$ & $\begin{array}{r}\text { Horz. } \\
\text { disp. }\end{array}$ & $\begin{array}{r}\text { Horz. } \\
\text { beta }\end{array}$ & $\begin{array}{r}\text { Vert. } \\
\text { beta }\end{array}$ & $\begin{array}{r}\text { Element } \\
\text { strength }\end{array}$ \\
& $\mathrm{N}$ & $\begin{array}{r}\lambda_{x} \\
{\left[\mathrm{~m}^{-2}\right]}\end{array}$ & $\begin{array}{r}\beta_{x} \\
{[\mathrm{~m}]}\end{array}$ & $\begin{array}{r}\beta_{y} \\
{[\mathrm{~m}]}\end{array}$ & $\left|\mathbf{k}_{i}\right|$ \\
\hline Arc dipole body & 144 & -0.0012 & 1.20 & 24.3 & 23.9 & 0.011 \\
Dipole lead end $F$ & 72 & -0.0064 & 1.65 & 39.7 & 12.8 & 0.076 \\
Dipole lead end $D$ & 72 & -0.0064 & 1.05 & 13.5 & 39.6 & 0.050 \\
Arc quad $F$ & 69 & -0.0007 & 1.77 & 49.7 & 9.7 & 0.008 \\
Arc quad $D$ & 69 & 0.0007 & 0.91 & 10.4 & 47.6 & 0.004 \\
\hline
\end{tabular}

\subsection{Skew sextupole harmonics in superconducting magnets}

Unwanted magnetic field harmonics in superconducting magnets, where the fields are "current dominated", are naturally larger than in normal conducting magnets, where fields are "iron dominated". Considerable care is necessary in the design of a superconducting dipole, for example, in order to reduce the unwanted allowed body harmonics - sextupole, decapole, et cetera - at all excitations. RHIC magnets of all kinds are well modeled by a body harmonic that is smoothly distributed along the length of a magnet, plus delta function end multipoles at the "lead" and "return" ends, where the conductor geometry is subject to mechanical (and other) constraints. (The coils are connected to the outside world at the lead end, but simply turn back into the magnet at the return end). For the most part the lead end multipoles are stronger than the return end multipoles.

Table 5 records the dominant systematic skew sextupole elements in RHIC. The body harmonic of a magnet is conveniently represented by an equivalent multipole kick at its center. Hence the optical functions assigned to the arc dipole correspond to the center of the dipole. There is a strong tendency for a set of systematic vectors in RHIC to cancel when they are added up, since the horizontal and vertical tunes differ by about one unit, and the element vectors tend to form a circle. This paper makes no attempt to properly and carefully add each set of systematic vectors. Rather, the last column in Table 5 simply lists $\left|\mathbf{k}_{i}\right|$, the strength of a single element. The arc dipole lead ends are clearly the dominant source of systematic skew sextupoles in RHIC.

Similarly, Table 6 records the dominant random skew sextupole elements in RHIC magnet harmonics. In this table it is trivial to estimate the total skew chromaticity strength due to a particular source, by multiplying the individual element strength by the square root of the element count. The last column lists the root mean square total strength, $<\mathbf{k}^{2}>^{1 / 2}$, averaged over an ensemble of accelerators. At the bottom of the table, the grand total of 0.40 records the sum of all random sources, added in quadrature. This is almost completely due to body harmonics in the arc dipoles and in the low beta interaction region (IR) quadrupoles.

Skew sextupole harmonics in RHIC magnets are less potent in generating skew chromaticity than vertical dispersion errors in chromaticity sextupoles. 
TABLE 6: Dominant sources of RANDOM skew sextupole kicks in RHIC.

\begin{tabular}{|c|c|c|c|c|c|c|}
\hline Magnet & Count & $\begin{array}{r}\text { Skew } \\
\text { sext. } \\
\text { strength }\end{array}$ & $\begin{array}{c}\text { Horz. } \\
\text { disp. }\end{array}$ & $\begin{array}{l}\text { Horz. } \\
\text { beta }\end{array}$ & $\begin{array}{l}\text { Vert. } \\
\text { beta }\end{array}$ & $\begin{array}{r}\text { TOTAL } \\
\text { strength } \\
(\mathrm{rms})\end{array}$ \\
\hline & $\mathrm{N}$ & $\begin{array}{r}A_{2} \\
{\left[\mathrm{~m}^{-} 2\right]}\end{array}$ & $\begin{array}{r}\eta_{x} \\
{[\mathrm{~m}]}\end{array}$ & $\begin{array}{r}\beta_{x} \\
{[\mathrm{~m}]}\end{array}$ & $\begin{array}{r}\beta_{y} \\
{[\mathrm{~m}]}\end{array}$ & $\left|\mathbf{k}_{i}\right|$ \\
\hline Arc dipole body & 144 & 0.0012 & 1.20 & 24.3 & 23.9 & 0.14 \\
\hline Dipole lead end $F$ & 72 & 0.0001 & 1.65 & 39.7 & 12.8 & 0.01 \\
\hline Dipole lead end $D$ & 72 & 0.0001 & 1.05 & 13.5 & 39.6 & 0.01 \\
\hline Arc quad $F$ & 69 & 0.0008 & 1.77 & 49.7 & 9.7 & 0.08 \\
\hline Arc quad $D$ & 69 & 0.0008 & 0.91 & 10.4 & 47.6 & 0.04 \\
\hline IR quad $1 F$ body & 2 & 0.00032 & -0.49 & 667 & 708 & 0.05 \\
\hline IR quad $1 D$ body & 2 & 0.00032 & 0.52 & 718 & 668 & 0.05 \\
\hline IR quad $2 F$ body & 2 & 0.00073 & 0.73 & 1354 & 550 & 0.21 \\
\hline IR quad $2 D$ body & 2 & 0.00073 & -0.47 & 558 & 1335 & 0.13 \\
\hline IR quad $3 F$ body & 2 & 0.00045 & -0.75 & 1332 & 556 & 0.13 \\
\hline IR quad $3 D$ body & 2 & 0.00045 & 0.49 & 575 & 1313 & 0.09 \\
\hline IR Q $1 F$ lead end & 2 & 0.00036 & -0.49 & 665 & 812 & 0.06 \\
\hline IR Q $1 D$ lead end & 2 & 0.00036 & 0.56 & 825 & 645 & 0.07 \\
\hline IR Q $2 F$ lead end & 2 & 0.00036 & 0.67 & 1127 & 743 & 0.10 \\
\hline IR Q $2 D$ lead end & 2 & 0.00036 & -0.55 & 754 & 1110 & 0.08 \\
\hline IR Q $3 F$ lead end & 2 & 0.00036 & -0.69 & 1151 & 715 & 0.10 \\
\hline IR Q $3 D$ lead end & 2 & 0.00036 & 0.54 & 725 & 1134 & 0.08 \\
\hline GRAND TOTAL & & & & & & 0.40 \\
\hline
\end{tabular}

\section{SUMMARY AND CONCLUSIONS}

The standard vector model of on-momentum linear coupling has been extended to include off-momentum particles. In this model, the difference in eigentunes is given by the length of a 3-D vector that is parameterised by $\delta$, the off-momentum parameter, and $\mathbf{k}$, the 2-D "skew chromaticity" vector. The values of the eigenchromaticities - defined as the differentials of the eigentunes with respect to $\delta$ - have been shown to be important in determining head-tail stability. Strong criteria on the normal chromaticities have been derived that guarantee headtail stability for any skew quadrupole and erect quadrupole settings. The off-momentum model and the new criteria are in good agreement with Tevatron experiments and with simple simulations.

The most potent source of skew chromaticity appears to be vertical dispersion in chromaticity sextupoles. Large machines are more vulnerable than small ones. All reasonable 
attempts must be made to keep the vertical dispersion in the arcs small. Accidental systematic and random harmonics in superconducting magnets can also contribute significantly to the net skew chromaticity vector. However, RHIC statistics suggest that, with care, these sources can be well controlled. Rolled chromaticity sextupoles are only a weak source of skew chromaticity.

\section{ACKNOWLEDGEMENTS}

We would like to thank several people who directly or indirectly supported the work of this paper. At the Fermi National Accelerator Laboratory, Gerry Annala, Glenn Goderre and several members of the Fermilab operations group participated in the 1990 Tevatron machine studies that first quantified the interaction of head-tail and linear coupling effects which, until then, had taken on the aura of mysterious mythology. Pat Colestock (and the RHIC Accelerator Advisory Committee) later encouraged us to return to a more systematic study of skew chromaticity phenomena. Tom Pelaia, Stu Peck, and Dave Rubin of the Cornell Electron Storage Ring helped in arranging to take the 1994 data that show that CESR is relatively immune to skew chromaticity problems. Thank you, everybody.

\section{REFERENCES}

1. D. Edwards and L. Teng, "Parameterization of Linear Coupled Motion in Periodic Systems", IEEE Trans. Nucl. Sci., Vol. NS-20, No. 3, (1973).

2. S. Peggs, "Coupling and Decoupling in Storage Rings", IEEE Trans. Nucl. Sci., Vol. NS-30, No. 4, Santa $\mathrm{Fe},(1983)$ p. 2460.

3. L. Schachinger and R. Talman, Manual for the Program TEAPOT, (Cornell University, 1994) Appendix F.

4. S. Peggs, "Head-Tail Stability and Linear Coupling”, Fermilab AP Note 90-007 (1990).

5. G.P. Goderre et al., "Head-Tail Stability and Linear Coupling in the Tevatron", IEEE Particle Accelerator Conference, San Francisco (1991) p. 1848.

6. Cornell Electron Storage Ring log book 116, unpublished (1994) pp. 123-125.

7. G.F. Dell and S. Peggs. TEAPOT tracking studies of RHIC were performed with the RHIC92.0.4 lattice tuned for luminosity conditions, with $\beta^{*}=1.0$ meter at two collision points. The MAC 94.2 standard set of magnetic and alignment errors were used. Unpublished (1994).

8. A. Chao, Physics of Collective Beam Instabilities in High Energy Accelerators (John Wiley and Sons, New York, 1993).

9. D. Edwards and M. Syphers, An Introduction to the Physics of High Energy Accelerators (John Wiley and Sons, New York, 1993).

10. R. Kohaupt, DESY Report M-80/19 (1980).

11. R. Talman, CERN Report ISR-TH/81-17 (1981); R. Talman, Nucl. Instr. Meth. 193, 423, (1982). 